\title{
Pacific
}

Journal of

Mathematics

\section{ODD HAMILTONIAN SUPERALGEBRAS AND SPECIAL ODD HAMILTONIAN SUPERALGEBRAS OF FORMAL VECTOR FIELDS}

Li Ren, QIANG Mu and YongZheng Zhang 


\title{
ODD HAMILTONIAN SUPERALGEBRAS AND SPECIAL ODD HAMILTONIAN SUPERALGEBRAS OF FORMAL VECTOR FIELDS
}

\author{
Li REN, QIANG Mu AND YongZheng ZHANG
}

\begin{abstract}
The natural filtrations of odd Hamiltonian superalgebras and special odd Hamiltonian superalgebras of formal vector fields are proved to be invariant under their automorphism group respectively, by determining the set of ad-quasi-nilpotent elements. Thereby, the automorphism groups of these Lie superalgebras are determined.
\end{abstract}

\section{Introduction}

As is well known, filtration structures and automorphism groups play an important role in the classification of modular Lie algebras (see [Jin 1992; Strade and Farnsteiner 1988; Wilson 1975]) and nonmodular Lie superalgebras (see [Kac 1977; Kac 1998; Scheunert 1979]), respectively. Cartan-type Lie algebras and Lie superalgebras possess natural filtration structures. The natural filtrations of the infinite-dimensional Lie algebras $L(m)$ and $\hat{L}(m)$ were proved to be invariant in [Rudakov 1986], where $L=W, S, H$ or $K$. The natural filtrations of the general Lie superalgebra and special Lie superalgebra of formal vector fields were proved to be invariant in [Zhang and Liu 2004]. The invariance of natural filtrations of Cartantype Lie algebras or Lie superalgebras provides a useful method of determining intrinsic properties and automorphism groups (see [Wilson 1971; Zhang and Liu 2004]).

We consider the infinite-dimensional odd Hamiltonian superalgebra $\mathrm{HO}$ and special odd Hamiltonian superalgebra $\mathrm{SHO}$ of formal vector fields, which are involved in [Kac 1998]. The corresponding results of Cartan-type Lie algebras are generalized and Jin's methods are used (see [Jin 1992]). Denote by $\left\{X_{i}\right\}_{i \geq-1}$

Qiang $\mathrm{Mu}$ is the corresponding author.

Supported by the Tianyuan Fund for Mathematics (grant number 11126051), the Heilongjiang Provincial Natural Science Foundation of China (grant number A201008), the Scientific Research Fund of Heilongjiang Provincial Education Department (grant number 12511157), and the Doctoral Research Foundation of Harbin Normal University (grant number KJB201105).

MSC2010: 17B40, 17B65, 17B66.

Keywords: superalgebra of formal vector fields, ad-nilpotent element, filtration, continuous automorphism. 
the natural filtration of $X$. By determining the ad-quasi-nilpotent elements in the even part and the subalgebras generated by certain ad-quasi-nilpotent elements, we prove that the natural filtration of $X$ is invariant under automorphisms in the following sense: If $\varphi$ is an automorphism of $X$, then $\varphi\left(X_{i}\right) \subseteq X_{i}$ for every $i \geq-1$. Besides, we prove that every automorphism of $X$ is continuous and can be induced from an automorphism of $\Lambda(n, n)$. Finally, we prove that the automorphism group of $X$ is isomorphic to the admissible automorphism group of the base superalgebra $\Lambda(n, n)$.

This paper is arranged as follows. In Section 2, we recall the necessary definitions concerning Lie superalgebras of Cartan type $\mathrm{HO}$ and $\mathrm{SHO}$ of formal vector fields. In Section 3, we characterize the ad-quasi-nilpotent elements with certain properties, and prove the invariance of their natural filtrations. In Section 4, we determine the automorphism groups of Lie superalgebras of Cartan type $H O$ and $\mathrm{SHO}$ of formal vector fields.

\section{Preliminaries}

In this paper, $\mathbb{F}$ denotes an algebraically closed field of characteristic zero, and $n$ is a positive integer greater than 3 . Let $\mathbb{N}$ and $\mathbb{N}_{0}$ denote the sets of positive integers and nonnegative integers, respectively. Let $\mathbb{Z}_{2}=\{\overline{0}, \overline{1}\}$ denote the ring of integers modulo 2. Let $\mathrm{P}(n)=\mathbb{F} \llbracket x_{1}, \ldots, x_{n} \rrbracket$ denote the ring of formal power series in $n$ variables over field $\mathbb{F}$. For $\alpha=\left(\alpha_{1}, \alpha_{2}, \ldots, \alpha_{n}\right) \in \mathbb{N}_{0}^{n}$, we abbreviate $x_{1}^{\alpha_{1}} x_{2}^{\alpha_{2}} \cdots x_{n}^{\alpha_{n}}$ to $x^{(\alpha)}$, and put $|\alpha|=\sum_{i=1}^{n} \alpha_{i}$. Let $\Lambda(n)$ be the Grassmann algebra over $\mathbb{F}$ in $n$ variables $x_{n+1}, x_{n+2}, \ldots, x_{2 n}$. Denote by $\Lambda(n, n)$ the tensor product $\mathrm{P}(n) \otimes_{\mathbb{F}} \Lambda(n)$. Then $\Lambda(n, n)$ is a noncommutative linearly compact topological superalgebra with a fundamental system $\left\{\left(\Lambda_{1}\right)^{j}\right\}_{j \geq 1}$ of neighborhoods of 0 , where $\left(\Lambda_{1}\right)^{j}=\operatorname{span}_{\mathbb{F}}\left\{x_{i_{1}} \cdots x_{i_{k}} \mid j \leq k\right\}$. In particular, $\left(\Lambda_{1}\right)^{1}$ is the ideal of $\Lambda(n, n)$ generated by $\left\{x_{1}, \ldots, x_{2 n}\right\}$ (see [Kac 1998]). For $g \in \mathrm{P}(n)$ and $f \in \Lambda(n)$, we abbreviate $g \otimes f$ to $g f$.

$$
\begin{array}{r}
\text { Put } Y_{0}=\{1,2, \ldots, n\}, Y_{1}=\{n+1, \ldots, 2 n\} \text { and } Y=Y_{0} \cup Y_{1} \text {. Let } \\
\qquad \mathbb{B}_{k}=\left\{\left\langle i_{1}, i_{2}, \ldots, i_{k}\right\rangle \mid n+1 \leq i_{1}<i_{2}<\cdots<i_{k} \leq 2 n\right\}
\end{array}
$$

and $\mathbb{B}(n)=\bigcup_{k=0}^{n} \mathbb{B}_{k}$, where $\mathbb{B}_{0}=\varnothing$. Given $u=\left\langle i_{1}, i_{2}, \ldots, i_{k}\right\rangle \in \mathbb{B}_{k}$, set $|u|=k$, $\{u\}=\left\{i_{1}, i_{2}, \ldots, i_{k}\right\}$ and $x^{u}=x_{i_{1}} x_{i_{2}} \cdots x_{i_{k}}$ (with the convention that $|\varnothing|=0$ and $\left.x^{\varnothing}=1\right)$. Then $\left\{x^{(\alpha)} x^{u} \mid \alpha \in \mathbb{N}_{0}^{n}, u \in \mathbb{B}(n)\right\}$ is an $\mathbb{F}$-basis of the infinitedimensional superalgebra $\Lambda(n, n)$. Clearly, $\Lambda(n, n)$ has a $\mathbb{Z}$-grading structure $\Lambda(n, n)=\bigoplus_{i \geq 0} \Lambda(n, n)_{[i]}$, where

$$
\Lambda(n, n)_{[i]}=\operatorname{span}_{\mathbb{F}}\left\{x^{(\alpha)} x^{u}|| \alpha|+| u \mid=i, \alpha \in \mathbb{N}_{0}^{n}, u \in \mathbb{B}(n)\right\} .
$$


An arbitrary element $f \in \Lambda(n, n)$ can be uniquely decomposed into $f=\sum_{i=0}^{\infty} f_{i}$, where $f_{i} \in \Lambda(n, n)_{[i]}$. The continuation of the addition of topological algebra $\Lambda(n, n)$ allows us to get the sum of infinite nonzero elements of $\Lambda(n, n)$. Set $\Lambda(n, n)_{j}=\bigoplus_{i \geq j} \Lambda(n, n)_{[i]}$. Then $\left\{\Lambda(n, n)_{j}\right\}_{j \geq 0}$ is a filtration of $\Lambda(n, n)$. Clearly, $\Lambda(n, n)_{j}=\left\{\left(\Lambda_{1}\right)^{j}\right\}$, where $j \in \mathbb{N}_{0}$.

Let $D_{1}, D_{2}, \ldots, D_{2 n}$ be the linear transformations of $\Lambda(n, n)$ such that

$$
D_{i}\left(x^{(\alpha)} x^{u}\right)= \begin{cases}x^{\left(\alpha-\varepsilon_{i}\right)} x^{u} & \text { if } i \in Y_{0}, \\ x^{(\alpha)} \cdot\left(\partial x^{u} / \partial x_{i}\right) & \text { if } i \in Y_{1} .\end{cases}
$$

Then $D_{i}$ is a derivation of superalgebra $\Lambda(n, n)$ for every $i \in Y$. Let $\operatorname{Der} \Lambda(n, n)$ be the Lie superalgebra consisting of all continuous derivations of $\Lambda(n, n)$. Then $\operatorname{Der} \Lambda(n, n)=W(n, n)$, where $W(n, n)=\left\{\sum_{i=1}^{2 n} f_{i} D_{i} \mid f_{i} \in \Lambda(n, n)\right\}$, and we call $W(n, n)$ the general superalgebra of formal vector fields (see [Kac 1998]). Clearly, $W(n, n)$ has a $\mathbb{Z}$-grading structure $W(n, n)=\bigoplus_{i \geq-1} W(n, n)_{[i]}$, where $W(n, n)_{[i]}=\operatorname{span}_{\mathbb{F}}\left\{f D_{j} \mid f \in \Lambda(n, n)_{[i+1]}, j \in Y\right\}$. Let

$$
W(n, n)_{j}=\bigoplus_{i \geq j} W(n, n)_{[i]} .
$$

Then $\left\{W(n, n)_{j}\right\}_{j \geq-1}$ is called the natural filtration of $W(n, n)$. Therefore, $W(n, n)$ is a linearly compact topological Lie superalgebra with $\left\{W(n, n)_{j}\right\}_{j \geq-1}$ as a fundamental system of neighborhoods of 0 .

If $\operatorname{deg} f$ appears in some expression in this paper, we always regard $f$ as a $\mathbb{Z}_{2}$-homogenous element and $\operatorname{deg} f$ as the $\mathbb{Z}_{2}$-degree of $f$. Then $\operatorname{deg} D_{i}=\mu(i)$, where

$$
\mu(i)= \begin{cases}\overline{0} & \text { if } i \in Y_{0}, \\ \overline{1} & \text { if } i \in Y_{1} .\end{cases}
$$

The following formula holds in $W(n, n)$ (see [Zhang 1997]):

$$
\left[f D_{i}, g D_{j}\right]=f D_{i}(g) D_{j}-(-1)^{\operatorname{deg} f D_{i} \operatorname{deg} g D_{j}} g D_{j}(f) D_{i},
$$

where $f, g \in \Lambda(n, n)$ and $i, j \in Y$.

Put

$$
i^{\prime}= \begin{cases}i+n & \text { if } i \in Y_{0}, \\ i-n & \text { if } i \in Y_{1} .\end{cases}
$$

Let $T_{H}: \Lambda(n, n) \rightarrow W(n, n)$ be the linear mapping such that

$$
T_{H}(f)=\sum_{i=1}^{2 n}(-1)^{\mu(i) \operatorname{deg} f} D_{i}(f) D_{i^{\prime}}
$$

Put $H O(n)=\left\{T_{H}(f) \mid f \in \Lambda(n, n)\right\}$. Then $H O(n)$ is an infinite-dimensional Lie superalgebra (see [Kac 1998]), called the odd Hamiltonian superalgebra of formal 
vector fields. For $f, g \in \Lambda(n, n)$ the equation

$$
\left[T_{H}(f), T_{H}(g)\right]=T_{H}\left(T_{H}(f)(g)\right)
$$

holds (see [Kac 1998]). Clearly, the algebra $H O(n)$ has a $\mathbb{Z}$-grading structure $H O(n)=\bigoplus_{i \geq-1} H O(n)_{[i]}$, where $H O(n)_{[i]}=\left\{T_{H}(f) \mid f \in \Lambda(n, n)_{[i+2]}\right\}$. Set $H O(n)_{i}=H O(n) \cap W(n, n)_{i}$. Then $\left\{H O(n)_{i}\right\}_{i \geq-1}$ is called the natural filtration of $H O(n)$.

Let $H O(n, n)$ be the $\mathbb{Z}_{2}$-graded space $\Lambda(n, n) / \mathbb{F} \cdot 1$ with reversed parity, that is, $H O(n, n)=H O(n, n)_{\overline{0}}+H O(n, n)_{\overline{1}}$, where

$$
H O(n, n)_{\theta}=\operatorname{span}_{\mathbb{F}}\left\{x^{(\alpha)} x^{u} \in \Lambda(n, n)_{\theta+\overline{1}}|| \alpha|+| u \mid \geq 1\right\}, \quad \theta \in \mathbb{Z}_{2} .
$$

We denote by $\mathrm{p}(y)$ the $\mathbb{Z}_{2}$-degree of the element $y$ of $H O(n, n)$ to distinguish it from the $\mathbb{Z}_{2}$-degree in $\Lambda(n, n)$. By (2), we can define a Lie multiplication in $H O(n, n)$ by

$$
[y, z]=\sum_{i=1}^{2 n}(-1)^{\mu(i) \mathrm{p}(y)+\mu(i)} D_{i}(y) D_{i^{\prime}}(z) .
$$

Clearly, Lie superalgebra $H O(n, n)$ is isomorphic to $H O(n)$.

Let $\Delta=\sum_{i=1}^{n} D_{i} D_{i^{\prime}}$ be a linear mapping on $\Lambda(n, n)$, let

$$
\Lambda^{\Delta}(n, n)=\{f \in \Lambda(n, n) \mid \Delta f=0\},
$$

and let $\overline{S H O}(n, n)=\Lambda^{\Delta}(n, n) / \mathbb{F} \cdot 1$. Then $\overline{\operatorname{SHO}}(n, n)$ is a $\mathbb{Z}_{2}$-graded subspace of $H O(n, n)$. For $f, g \in \Lambda(n, n)$ we have

$$
\Delta\left(T_{H}(f)(g)\right)=(-1)^{\operatorname{deg} f+\overline{1}} T_{H}(f)(\Delta g)-(-1)^{\operatorname{deg} f \operatorname{deg} g+\operatorname{deg} f} T_{H}(g)(\Delta f) ;
$$

see [Kac 1998]. Therefore, with the multiplication defined in (3), $\overline{\operatorname{SHO}}(n, n)$ is a subalgebra of $H O(n, n)$. Set

$$
\operatorname{SHO}(n, n)=\operatorname{span}_{\mathbb{F}}\left\{\left[x^{(\alpha)}, x^{u}\right]\left|\alpha \in \mathbb{N}_{0}^{n}, u \in \mathbb{B}(n),\right| \alpha|+| u \mid \geq 3\right\} .
$$

Then $\operatorname{SHO}(n, n)$ is an infinite-dimensional subalgebra of $\overline{\operatorname{SHO}}(n, n)$, called the special odd Hamiltonian superalgebra of formal vector fields (see [Kac 1998]). Clearly, $\operatorname{SHO}(n, n)$ has a $\mathbb{Z}$-grading structure $\operatorname{SHO}(n, n)=\bigoplus_{i \geq-1} \operatorname{SHO}(n, n)_{[i]}$, where

$$
\operatorname{SHO}(n, n)_{[i]}=\operatorname{span}_{\mathbb{E}}\left\{\left[x^{(\alpha)}, x^{u}\right]\left|\alpha \in \mathbb{N}_{0}^{n}, u \in \mathbb{B}(n),\right| \alpha|+| u \mid=i+4\right\} .
$$

Set $\operatorname{SHO}(n, n)_{i}=\operatorname{SHO}(n, n) \cap W(n, n)_{i}$. Then $\left\{\operatorname{SHO}(n, n)_{i}\right\}_{i \geq-1}$ is called the natural filtration of $\operatorname{SHO}(n, n)$.

Set $\operatorname{SHO}(n)=T_{H}(\operatorname{SHO}(n, n))$. Clearly, with the multiplication defined in (2), $\operatorname{SHO}(n)$ is a Lie superalgebra that is isomorphic to $\operatorname{SHO}(n, n)$. For the sake of simplicity, we always write $\mathrm{SHO}$ for $\mathrm{SHO}(n, n)$ or $\operatorname{SHO}(n)$. 
In the following, we simply write $\mathrm{HO}$ for $H O(n)$, and let $\mathrm{X}$ denote the Lie superalgebra $\mathrm{HO}$ or $\mathrm{SHO}$.

\section{Invariant subalgebras and natural filtrations}

Lemma 3.1. Suppose that $y \in X_{[-1]} \cap X_{\overline{0}}$ and that $y \neq 0$. Then

$$
y\left(\Lambda(n, n)_{[r]}\right)=\Lambda(n, n)_{[r-1]}
$$

for all $r \in \mathbb{N}$; hence $y(\Lambda(n, n))=\Lambda(n, n)$.

Proof. We first prove that $\Lambda(n, n)_{[r-1]} \subseteq y\left(\Lambda(n, n)_{[r]}\right)$. Write $y=\sum_{j=1}^{n} c_{j} D_{j}$, where $c_{j} \in \mathbb{F}$. Then there exists at least one nonzero element in $\left\{c_{1}, \ldots, c_{n}\right\}$. Let $c_{1 j}=c_{j}$, where $1 \leq j \leq n$. Then there exist $c_{l j} \in \mathbb{F}$, where $2 \leq l \leq n, 1 \leq j \leq n$, such that the matrix $\left(c_{i j}\right)_{1 \leq i, j \leq n}$ is invertible. Let $\left(a_{i j}\right)_{1 \leq i, j \leq n}=\left(c_{i j}\right)_{1 \leq i, j \leq n}^{-1}$. Note that $(1,0, \ldots, 0)\left(c_{i j}\right)_{1 \leq i, j \leq n}=\left(c_{1}, c_{2}, \ldots, c_{n}\right)$. It follows that

$$
(1,0, \ldots, 0)=\left(c_{1}, c_{2}, \ldots, c_{n}\right)\left(a_{i j}\right)_{1 \leq i, j \leq n} .
$$

Let $h_{j}=\sum_{i=1}^{n} a_{i j} x_{i}$, where $j \in Y_{0}$, and let $h_{k}=x_{k}$, where $k \in Y_{1}$. Then the set $\left\{h_{1}, h_{2}, \ldots, h_{2 n}\right\}$ is an $\mathbb{F}$-basis of $\Lambda(n, n)_{[1]}$. Therefore, for every $r \in \mathbb{N}$, we have

$$
\Lambda(n, n)_{[r-1]}=\operatorname{span}_{\mathbb{F}}\left\{h_{1}^{\alpha_{1}} \cdots h_{n}^{\alpha_{n}} h_{i_{1}} \cdots h_{i_{k}}\right\},
$$

where $\left(\alpha_{1}, \ldots, \alpha_{n}\right) \in \mathbb{N}_{0}^{n},\left\langle i_{1}, \ldots, i_{k}\right\rangle \in \mathbb{B}_{k}$ and $\sum_{i=1}^{n} \alpha_{i}+k=r-1$. Noting that $\alpha_{1} \in \mathbb{N}_{0}$, we see that $\alpha_{1}+1 \neq 0$, since char $\mathbb{F}=0$. By (5), we have $y\left(h_{j}\right)=\delta_{j 1}$, where $j \in Y_{0}$. Consequently, we have

$$
y\left(\left(\alpha_{1}+1\right)^{-1} h_{1} h_{1}^{\alpha_{1}} \cdots h_{n}^{\alpha_{n}} h_{i_{1}} \cdots h_{i_{k}}\right)=h_{1}^{\alpha_{1}} \cdots h_{n}^{\alpha_{n}} h_{i_{1}} \cdots h_{i_{k}} .
$$

Thus $\Lambda(n, n)_{[r-1]} \subseteq y\left(\Lambda(n, n)_{[r]}\right)$. The reverse inclusion can be verified trivially.

Suppose that $f=\sum_{s \geq 0} f_{s}$ is an arbitrary element of $\Lambda(n, n)$, where $f_{s}$ is in $\Lambda(n, n)_{[s]}$. According to the results above, for every $s \in \mathbb{N}_{0}$, there exists a $g_{s+1}$ in $\Lambda(n, n)_{[s+1]}$ such that $f_{s}=y\left(g_{s+1}\right)$. Since $y$ is continuous, it follows that $f=\sum_{s \geq 0} y\left(g_{s+1}\right)=y\left(\sum_{s \geq 0} g_{s+1}\right) \in y(\Lambda(n, n))$. Thus $\Lambda(n, n)=y(\Lambda(n, n))$.

Lemma 3.2. Suppose that $y \in X_{[-1]} \cap X_{\overline{0}}$ and that $y \neq 0$. Then $\left[y, X_{[r]}\right]=X_{[r-1]}$ for all $r \in \mathbb{N}_{0}$.

Proof. It suffices to show that $X_{[r-1]} \subseteq\left[y, X_{[r]}\right]$. Consider the case of $H O$. Suppose that $T_{H}(f)$ is an element of $H O_{[r-1]}$, where $f \in \Lambda(n, n)_{[r+1]}$. Then by Lemma 3.1 there exists $g \in \Lambda(n, n)_{[r+2]}$ such that $y(g)=f$, which combined with (2) yields that $T_{H}(f)=\left[y, T_{H}(g)\right] \in\left[y, H O_{[r]}\right]$.

Consider the case of $S H O$. Suppose that $\left[x^{(\alpha)}, x^{u}\right]$ is a standard basis element of $S H O_{[r-1]}$, and assume that $y=x_{i^{\prime}}$, where $i \in Y_{0}$. Then by (3), we have that $-\left[x^{(\alpha)}, x^{u}\right]=\left[x_{i^{\prime}},\left[x^{\left(\alpha+\varepsilon_{i}\right)}, x^{u}\right]\right]$ is in $\left[y, S H O_{[r]}\right]$. 
Lemma 3.3. Suppose that $y \in X_{-1} \cap X_{\overline{0}} \backslash X_{0}$ and that $y \neq 0$. Then $[y, X]=X$.

Proof. It suffices to show that $X \subseteq[y, X]$. Suppose that $y=\sum_{i \geq-1} y_{i}$, where $y_{i} \in X_{[i]}$, and suppose that $z=\sum_{i \geq-1} z_{i}$ is an arbitrary element of $X$, where $z_{i} \in X_{[i]}$. Then by Lemma 3.2, we can inductively pick $w_{j} \in X_{[j]}$, such that $\left[y_{-1}, w_{0}\right]=z_{-1}$ when $j=0$, and $\left[y_{-1}, w_{j}\right]=z_{j-1}-\sum_{i=0}^{j-1}\left[y_{i}, w_{j-1-i}\right]$ when $j>0$. For arbitrary $k \in \mathbb{N}_{0}$, direct calculations show that

$$
\left[y_{-1}, \sum_{j=0}^{k} w_{j}\right]=\left[y_{-1}, w_{0}\right]+\sum_{j=1}^{k}\left[y_{-1}, w_{j}\right]=\sum_{j=-1}^{k-1} z_{j}-\sum_{0 \leq i+j \leq k-1}\left[y_{i}, w_{j}\right],
$$

and

$$
\begin{aligned}
& {\left[\sum_{i \geq 0} y_{i}, \sum_{j=0}^{k} w_{j}\right]=\left[\sum_{i=0}^{k-1} y_{i}, \sum_{j=0}^{k} w_{j}\right]+\left[\sum_{i \geq k} y_{i}, \sum_{j=0}^{k} w_{j}\right] } \\
&=\sum_{0 \leq i+j \leq k-1}\left[y_{i}, w_{j}\right]+\sum_{i+j \geq k}\left[y_{i}, w_{j}\right] .
\end{aligned}
$$

Combining (6) and (7), we have

$$
\begin{aligned}
{\left[y, \sum_{j=0}^{k} w_{j}\right]=\left[y_{-1}, \sum_{j=0}^{k} w_{j}\right]+\left[\sum_{i \geq 0} y_{i}\right.} & \left., \sum_{j=0}^{k} w_{j}\right] \\
& =\sum_{j=-1}^{k-1} z_{j}+\sum_{i+j \geq k}\left[y_{i}, w_{j}\right] \in \sum_{j=-1}^{k-1} z_{j}+X_{k} .
\end{aligned}
$$

Noting that $X_{k}=\bigcap_{i=0}^{k} X_{i}$, we see that $\left[y, \sum_{j=0}^{k} w_{j}\right] \equiv \sum_{j=-1}^{k-1} z_{j}\left(\bmod \bigcap_{i=0}^{k} X_{i}\right)$. Let $w=\sum_{j \geq 0} w_{j}$. Then $[y, w]=\left[y, \sum_{j \geq 0} w_{j}\right] \equiv \sum_{j \geq-1} z_{j}\left(\bmod \bigcap_{i \geq 0} X_{i}\right)$, whence $[y, w]=z$. Thus $X \subseteq[y, X]$.

For an element $y$ of Lie superalgebra $L$, we call $y$ ad-nilpotent if there exists a positive integer $t$ such that $(\operatorname{ad} y)^{t}(L)=0$. We call $y$ ad-quasi-nilpotent if $\bigcap_{t=1}^{\infty}(\operatorname{ad} y)^{t}(L)=0$ (see [Humphreys 1972; Jin 1992]). Obviously, ad-nilpotent elements are ad-quasi-nilpotent elements. In particular, $D_{i}$ is an ad-nilpotent element of $X$ for every $i \in Y_{1}$.

Let $J$ be a subset of $L$. Put

$$
\mathrm{qn}_{L}(J):=\{y \in J \mid y \text { is an ad-quasi-nilpotent element of } L\} .
$$

In the following, we simply write $\mathrm{qn}(J)$ for $\mathrm{qn}_{X}(J)$, and denote by $\mathrm{Qn}(J)$ the subalgebra of $X$ generated by qn $(J)$. It is clear that $X_{1} \subseteq \mathrm{qn}(X)$. In the following, we will determine the ad-quasi-nilpotent elements of $X_{[0]}$, and prove the invariance of natural filtration of $X$.

We denote by $\mathrm{M}_{2 n}(\Lambda(n, n))$ the $\mathbb{F}$-algebra consisting of all $2 n \times 2 n$ matrices over $\Lambda(n, n)$, denote by $\operatorname{pr}_{[0]}$ the projection of $\Lambda(n, n)$ on $\Lambda(n, n)_{[0]}$, and denote 
by $\operatorname{pr}_{1}$ the projection on $\Lambda(n, n)_{1}$. For $\left(a_{i j}\right)_{i, j \in Y} \in \mathrm{M}_{2 n}(\Lambda(n, n))$, we also denote

$$
\operatorname{pr}_{[0]}:\left(a_{i j}\right)_{i, j \in Y} \mapsto\left(\operatorname{pr}_{[0]}\left(a_{i j}\right)\right)_{i, j \in Y} \quad \text { and } \quad \operatorname{pr}_{1}:\left(a_{i j}\right)_{i, j \in Y} \mapsto\left(\operatorname{pr}_{1}\left(a_{i j}\right)\right)_{i, j \in Y}
$$

Lemma 3.4. Suppose that $h_{1}, h_{2}, \ldots, h_{2 n} \in \Lambda(n, n)_{1}$ with $\operatorname{deg}\left(h_{j}\right)=\mu(j)$ such that the matrix $\left(\operatorname{pr}_{[0]}\left(D_{i} h_{j}\right)\right)_{i, j \in Y}$ is invertible. Then there exists an automorphism $\sigma$ of $\Lambda(n, n)$ such that

$$
\sigma\left(x_{i}\right)=h_{i} \quad \text { for all } i \in Y .
$$

Proof. Let $\sigma: \Lambda(n, n) \rightarrow \Lambda(n, n)$ be an even endomorphism such that (8) holds. Note that the natural filtration of $\Lambda(n, n)$ is invariant under $\sigma$. Then $\sigma$ induces a linear transformation $\sigma_{i}$ of $\Lambda(n, n)_{i} / \Lambda(n, n)_{i+1}$ for every $i \geq 0$. We first use induction on $k$ to show that $\sigma_{k}$ is bijective. Since the matrix of $\sigma_{1}$ with respect to $\mathbb{F}$-basis $\left\{x_{1}+\Lambda(n, n)_{2}, \ldots, x_{2 n}+\Lambda(n, n)_{2}\right\}$ is just $\left(\operatorname{pr}_{[0]}\left(D_{i} h_{j}\right)\right)_{i, j \in Y}$, we see that $\sigma_{1}$ is bijective. Suppose that $k>1$ and $x^{(\alpha)} x^{u}$ is an element of $\Lambda(n, n)_{[k]}$. Then we can write $x^{(\alpha)} x^{u}=f_{j} f_{k-j}$, where $f_{j} \in \Lambda(n, n)_{[j]}$ and $f_{k-j} \in \Lambda(n, n)_{[k-j]}$ with $1 \leq j<k$. By induction, there exist $f_{j}^{\prime} \in \Lambda(n, n)_{[j]}$ and $f_{k-j}^{\prime} \in \Lambda(n, n)_{[k-j]}$ such that $\sigma\left(f_{j}^{\prime}\right) \equiv f_{j}\left(\bmod \Lambda(n, n)_{j+1}\right)$ and $\sigma\left(f_{k-j}^{\prime}\right) \equiv f_{k-j}\left(\bmod \Lambda(n, n)_{k-j+1}\right)$, whence

$$
\sigma\left(f_{j}^{\prime} f_{k-j}^{\prime}\right)=\sigma\left(f_{j}^{\prime}\right) \sigma\left(f_{k-j}^{\prime}\right) \equiv f_{j} f_{k-j}=x^{(\alpha)} x^{u}\left(\bmod \Lambda(n, n)_{k+1}\right) .
$$

Thus $\sigma_{k}$ is surjective. Note that since $\Lambda(n, n)_{k} / \Lambda(n, n)_{k+1}$ is finite-dimensional, it follows that $\sigma_{k}$ is bijective.

We next prove that $\sigma$ is bijective. Suppose that $f \in \operatorname{ker}(\sigma) \cap \Lambda(n, n)_{i}$ for any $i \geq 0$. Then $\sigma_{i}\left(f+\Lambda(n, n)_{i+1}\right)=0$. It follows that $f \in \Lambda(n, n)_{i+1}$, since $\sigma_{i}$ is injective. Thus $\operatorname{ker}(\sigma) \subseteq \bigcap_{j \geq i} \Lambda(n, n)_{j}=0$, and $\sigma$ is injective. Suppose that $g=g_{0}+g_{1} \in \Lambda(n, n)$, where $g_{0} \in \mathbb{F}, g_{1} \in \Lambda(n, n)_{1}$. Since $\sigma_{1}$ is surjective, there exists $g_{1}^{\prime} \in \Lambda(n, n)_{1}$ such that $\sigma_{1}\left(g_{1}^{\prime}+\Lambda(n, n)_{2}\right)=g_{1}+\Lambda(n, n)_{2}$. It follows that $g_{2}:=g_{1}-\sigma\left(g_{1}^{\prime}\right) \in \Lambda(n, n)_{2}$. Note that $\sigma_{i}$ is surjective for every $i \geq 0$. Then we can inductively pick $g_{i}^{\prime} \in \Lambda(n, n)_{i}$, and define $g_{i+1} \in \Lambda(n, n)_{i+1}$ by

$$
g_{i+1}:=g_{i}-\sigma\left(g_{i}^{\prime}\right) .
$$

Let $g^{\prime}=g_{0}+\sum_{i \geq 0} g_{i}^{\prime}$. Since $\sigma$ is continuous, it follows from (9) that

$$
\sigma\left(g^{\prime}\right)=\sigma\left(g_{0}\right)+\sum_{i \geq 0} \sigma\left(g_{i}^{\prime}\right)=g_{0}+\sum_{i \geq 1}\left(g_{i}-g_{i+1}\right)=g_{0}+g_{1}=g,
$$

whence $\sigma$ is surjective.

Let $\rho$ be the corresponding representation with respect to $X_{[0]}$-module $X_{[-1]}$, that is, $\rho(y)=\left.\operatorname{ad} y\right|_{X_{[-1]}}$ for all $y \in X_{[0]}$. It is easy to see that $\rho$ is faithful. For $y \in X_{[0]}$, we also denote by $\rho(y)$ the matrix of $\rho(y)$ relative to the fixed ordered 
$\mathbb{F}$-basis $\left\{D_{1}, D_{2}, \ldots, D_{2 n}\right\}$. Denote by $\mathrm{gl}(n, n)$ the general linear Lie superalgebra of $2 n \times 2 n$ matrices over $\mathbb{F}$ (see [Scheunert 1979]).

Lemma 3.5. Suppose that $A$ is an invertible matrix of $\operatorname{gl}(n, n)$, and $y \in W(n, n)_{[0]}$. Then there exists an automorphism $\varphi$ of $W(n, n)$ such that $\rho(\varphi(y))=A \rho(y) A^{-1}$.

Proof. Suppose that $A=\left(a_{i j}\right)_{1 \leq i, j \leq 2 n}$, and let $h_{j}=\sum_{i=1}^{2 n} a_{i j} x_{i}$, where $1 \leq j \leq 2 n$. Then $\left\{h_{1}, h_{2}, \ldots, h_{2 n}\right\}$ is an $\mathbb{F}$-basis of $\Lambda(n, n)_{[1]}$. Note that $D_{i}\left(h_{j}\right)=a_{i j}$ for all $i, j \in Y$. It follows from Lemma 3.4 that there exists $\sigma \in$ Aut $\Lambda(n, n)$ such that $\sigma\left(x_{i}\right)=h_{i}$ for all $i \in Y$. Clearly, $\sigma \in \operatorname{Aut}(\Lambda(n, n): W(n, n))$.

Let $\varphi: W(n, n) \rightarrow W(n, n)$ be the linear mapping such that $z \mapsto \sigma z \sigma^{-1}$ for all $z$ in $W(n, n)$. Then $\varphi$ is an automorphism of $W(n, n)$. We claim that $\varphi$ is the desired automorphism. Suppose that $A^{-1}=\left(c_{i j}\right)_{1 \leq i, j \leq 2 n}$, and let $y=\sum_{s, r \in Y} b_{s r} x_{s} D_{r}$ be an arbitrary element of $W(n, n)_{[0]}$, where $b_{s r} \in \mathbb{F}$. Then $\rho(y)=\left(b_{s r}\right)_{1 \leq s, r \leq 2 n}$. Noting that $(\varphi y)\left(\sigma x_{i}\right)=\sigma\left(y x_{i}\right)$, we see that $\varphi(y)=\sum_{t, j \in Y} \sigma\left(y x_{t}\right) D_{j}$. Thus

$$
\begin{aligned}
\varphi(y)=\sum_{t, j \in Y} c_{t j} \sigma\left(\sum_{s \in Y} b_{s t} x_{s}\right) D_{j}=\sum_{t, j, s \in Y} b_{s t} c_{t j} \sigma\left(x_{s}\right) D_{j} \\
\quad=\sum_{t, j, s \in Y} b_{s t} c_{t j} h_{s} D_{j}=\sum_{t, j, s, k \in Y} a_{k s} b_{s t} c_{t j} x_{k} D_{j} .
\end{aligned}
$$

It follows that $\rho(\varphi(y))=A \rho(y) A^{-1}$.

Lemma 3.6. Suppose that $y \in W(n, n)_{[0]}$. Then ad $y$ is a nilpotent linear transformation of $W(n, n)_{[r]}$ for every $r \geq-1$ if and only if $\rho(y)$ is a nilpotent matrix.

Proof. If ad $\left.y\right|_{W(n, n)_{[-1]}}$ is nilpotent, then the definition of $\rho$ shows that $\rho(y)$ is a nilpotent matrix. Conversely, suppose that $\rho(y)$ is nilpotent. By Lemma 3.5, it suffices to consider the case when $\rho(y)$ is a strictly upper triangular matrix. Suppose that $y=\sum_{i, j \in Y, i<j} a_{i j} x_{i} D_{j}$, where $a_{i j} \in \mathbb{F}$.

We first prove that ad $x_{i} D_{j}$ is nilpotent linear transformation of $W(n, n)_{[r]}$ for every $r \geq-1$ when $i<j$. For any standard basis element $x^{(\alpha)} x^{u} D_{k}$ of $W(n, n)_{[r]}$, where $\alpha \in \mathbb{N}_{0}^{n}, u \in \mathbb{B}(n)$ and $k \in Y$, two cases arise.

Case 1. $j \in Y_{0}$. If $k \neq i$, then

$$
\left(\operatorname{ad} x_{i} D_{j}\right)^{t}\left(x^{(\alpha)} x^{u} D_{k}\right)=x_{i}^{t} D_{j}^{t}\left(x^{(\alpha)}\right) x^{u} D_{k}=0
$$

when $t \geq r+2$. If $k=i$, then

$$
\left(\operatorname{ad} x_{i} D_{j}\right)^{t}\left(x^{(\alpha)} x^{u} D_{i}\right)=x_{i}^{t} D_{j}^{t}\left(x^{(\alpha)}\right) x^{u} D_{i}-t x_{i}^{t-1} D_{j}^{t-1}\left(x^{(\alpha)}\right) x^{u} D_{j}=0
$$

when $t \geq r+3$.

Case 2. $j \in Y_{1}$. If $k \neq i$, then

$$
\left(\operatorname{ad} x_{i} D_{j}\right)^{t}\left(x^{(\alpha)} x^{u} D_{k}\right)=\left(\operatorname{ad} x_{i} D_{j}\right)^{t-2}\left(x_{i}^{2} x^{(\alpha)} D_{j}^{2}\left(x^{u}\right) D_{k}\right)=0
$$


when $t \geq 2$, and if $k=i$, then

$$
\left(\operatorname{ad} x_{i} D_{j}\right)^{t}\left(x^{(\alpha)} x^{u} D_{i}\right)=l\left(\operatorname{ad} x_{i} D_{j}\right)^{t-3}\left(x_{i}^{2} x^{(\alpha)} D_{j}^{2}\left(x^{u}\right) D_{j}\right)=0
$$

when $t \geq 3$, where $l=1$ or $l=-1$.

Therefore $\left(\operatorname{ad} x_{i} D_{j}\right)^{t}\left(x^{(\alpha)} x^{u} D_{k}\right)=0$ when $t \geq r+4$. Let $f_{k}=\sum_{\alpha, u} c_{\alpha, u} x^{(\alpha)} x^{u}$ be an arbitrary element of $\Lambda(n, n)_{[r+1]}$, where $c_{\alpha, u} \in \mathbb{F}, k \in Y$. Then for any $t \geq r+4$

$$
\left(\operatorname{ad} x_{i} D_{j}\right)^{t}\left(f_{k} D_{k}\right)=\sum_{\alpha, u}\left(\operatorname{ad} x_{i} D_{j}\right)^{t}\left(c_{\alpha, u} x^{(\alpha)} x^{u} D_{k}\right)=0,
$$

since $\left.\left(\operatorname{ad} x_{i} D_{j}\right)\right)^{t}$ is continuous. Consequently, we see that ad $\left.x_{i} D_{j}\right|_{W(n, n)_{[r]}}$ is nilpotent for every $r \geq-1$ when $i<j$.

Note that the set $\left\{ \pm x_{i} D_{j}, 0 \mid i<j\right\}$ is closed under the multiplication of $W(n, n)$, and the Lie superalgebra $\operatorname{span}_{\mathbb{F}}\left\{ \pm x_{i} D_{j}, 0 \mid i<j\right\}$ is finite-dimensional. It follows from [Strade and Farnsteiner 1988, Theorem 1.3.1] that ad $\left.y\right|_{W(n, n)_{[r]}}$ is nilpotent.

Lemma 3.7. Suppose that $y \in X_{[0]}$. Then ad $y$ is a nilpotent linear transformation of $X_{[r]}$ for every $r \geq-1$ if and only if $\rho(y)$ is a nilpotent matrix.

Proof. Clearly, $\rho(y)$ is a nilpotent matrix when ad $\left.y\right|_{X_{[-1]}}$ is nilpotent. Conversely, suppose that $\rho(y)$ is a nilpotent matrix. Then by Lemma 3.6, ad $y$ is a nilpotent linear transformation of $W(n, n)_{[r]}$ for every $r \geq-1$. Since $X$ is a subalgebra of $W(n, n)$, it follows that ad $\left.y\right|_{X_{[r]}}$ is nilpotent.

Lemma 3.8. Suppose that $y=y_{0}+y_{1} \in \mathrm{qn}\left(X_{0}\right)$, where $y_{0} \in X_{[0]}, y_{1} \in X_{1}$. Then $\rho\left(y_{0}\right)$ is a nilpotent matrix, and hence, $y_{0} \in \mathrm{qn}\left(X_{[0]}\right)$.

Proof. Let $X_{(i)}=X / X_{i+1}$ for every $i \geq-1$. Then $X_{(i)} \cong \bigoplus_{j \leq i} X_{[j]}$. For every $i \geq-1$, let $\tau_{i}$ be the endomorphism on $X_{(i)}$ satisfying $\tau_{i}(z) \equiv[y, z]\left(\bmod X_{i+1}\right)$ for all $z \in X_{(i)}$. Assume that $\rho\left(y_{0}\right)$ is not a nilpotent matrix. Then $\tau_{i}$ is not nilpotent for every $i \geq-1$. Let $X_{(i)}=U_{i} \oplus V_{i}$ be the Fitting decomposition of $X_{(i)}$ with respect to $\tau_{i}$, where $U_{i} \neq 0,\left.\tau_{i}\right|_{U_{i}}$ is invertible, $\left.\tau_{i}\right|_{V_{i}}$ is nilpotent. Since $X_{(i)}=X_{(i+1)} / X_{[i+1]}$, $\tau_{i}=\tau_{i+1}\left(\bmod X_{i+1}\right)$ and $\tau_{i+1}\left(X_{[i+1]}\right) \subseteq\left(X_{[i+1]}\right)$, it follows that

$$
X_{(i)}=\left(U_{i+1}+X_{[i+1]} / X_{[i+1]}\right) \oplus\left(V_{i+1}+X_{[i+1]} / X_{[i+1]}\right) .
$$

is also the Fitting decomposition of $X_{(i)}$ with respect to $\tau_{i}$, and by the uniqueness of the Fitting decomposition, we get $U_{i}=U_{i+1}+X_{[i+1]} / X_{[i+1]}$. This implies that $U_{i}$ is the projection of $U_{i+1}$ on $X_{(i)}$. Set

$$
\bar{U}=\left\{z \in X \mid \text { the projection of } z \text { on } X_{(i)} \text { belongs to } U_{i} \text { for all } i \geq-1\right\} .
$$

By the completeness of $X$, the set $\bar{U}$ is nonempty, and its projection on $X_{(i)}$ is $U_{i}$ for each $i \geq-1$. It follows that $[y, \bar{U}]=\bar{U}$. So $\bigcap_{t=0}^{\infty}(\text { ad } y)^{t}(X) \supseteq \bar{U} \neq 0$, 
contradicting the hypothesis that $y$ is ad-quasi-nilpotent. Therefore, $\rho\left(y_{0}\right)$ is a nilpotent matrix, which combined with Lemma 3.7 yields $y_{0} \in \mathrm{qn}\left(X_{[0]}\right)$.

Proposition 3.9. One has $\mathrm{qn}\left(X_{0}\right)=A N_{0} \cap X_{[0]}+X_{1}$, where

$$
A N_{0}=\left\{y \mid y \in W(n, n)_{[0]} \text { such that } \rho(y) \text { is a nilpotent matrix }\right\} .
$$

Proof. By Lemma 3.8, it suffices to show that $A N_{0} \cap X_{[0]}+X_{1} \subseteq \mathrm{qn}\left(X_{0}\right)$. Suppose that $y_{0} \in A N_{0} \cap X_{[0]}$, and suppose that $y_{1} \in X_{1}$. Let $y=y_{0}+y_{1}$. Then $\rho\left(y_{0}\right)$ is a nilpotent matrix. According to Lemma 3.7, there exists a positive integer $t_{i}$ such that $\left(\operatorname{ad} y_{0}\right)^{t_{i}}\left(X_{[i]}\right)=0$ for each $i \geq-1$. This implies that $\left(\operatorname{ad} y_{0}\right)^{t_{i}}\left(X_{i}\right) \subseteq X_{i+1}$. Consequently, we have $(\operatorname{ad} y)^{t_{-1}+\cdots+t_{k}}\left(X_{0}\right) \subseteq X_{k+1}$ for any $k \geq-1$. It follows that $\bigcap_{t=1}^{\infty}(\operatorname{ad} y)^{t}\left(X_{0}\right) \subseteq \bigcap_{k=1}^{\infty} X_{k}=0$, whence $y \in \mathrm{qn}\left(X_{0}\right)$.

Lemma 3.10. $\operatorname{Qn}\left(X_{\overline{0}}\right)=\operatorname{Qn}\left(X_{[0]} \cap X_{\overline{0}}\right)+X_{1} \cap X_{\overline{0}}$, and then $\operatorname{Qn}\left(X_{\overline{0}}\right) \subseteq X_{0} \cap X_{\overline{0}}$.

Proof. Note that $X_{1} \subseteq \mathrm{Qn}(X)$. It follows that $X_{1} \cap X_{\overline{0}} \subseteq \mathrm{Qn}\left(X_{\overline{0}}\right)$. Consequently, we have $\operatorname{Qn}\left(X_{[0]} \cap X_{\overline{0}}\right)+X_{1} \cap X_{\overline{0}} \subseteq \operatorname{Qn}\left(X_{\overline{0}}\right)$.

Conversely, suppose that $y=y_{-1}+y_{0} \in \mathrm{qn}\left(X_{\overline{0}}\right)$, where $y_{-1} \in X_{[-1]} \cap X_{\overline{0}}$, and $y_{0} \in X_{0} \cap X_{\overline{0}}$. Assume that $y_{-1} \neq 0$. It follows from Lemma 3.3 that $[y, X]=X$, which implies that $y$ is not an ad-quasi-nilpotent element of $X$, a contradiction. Thus $y \in X_{0} \cap X_{\overline{0}}$.

Now we can write $y=y_{0}+y_{1}$, where $y_{0} \in X_{[0]} \cap X_{\overline{0}}$, and $y_{1} \in X_{1} \cap X_{\overline{0}}$. By Lemma 3.8, we have $y_{0} \in \operatorname{qn}\left(X_{[0]} \cap X_{\overline{0}}\right) \subseteq \operatorname{Qn}\left(X_{[0]} \cap X_{\overline{0}}\right)$. It follows that $y \in \operatorname{Qn}\left(X_{[0]} \cap X_{\overline{0}}\right)+X_{1} \cap X_{\overline{0}}$. Thus

$$
\mathrm{qn}\left(X_{\overline{0}}\right) \subset \mathrm{Qn}\left(X_{[0]} \cap X_{\overline{0}}\right)+X_{1} \cap X_{\overline{0}} .
$$

The right-hand side of (10) is a subalgebra of $X_{\overline{0}}$. Then

$$
\operatorname{Qn}\left(X_{\overline{0}}\right) \subset \operatorname{Qn}\left(X_{[0]} \cap X_{\overline{0}}\right)+X_{1} \cap X_{\overline{0}} .
$$

Thus the lemma holds.

Lemma 3.11. Suppose that $i, j \in Y$ with $i \neq j^{\prime}$. Then $T_{H}\left(x_{i} x_{j}\right) \in \mathrm{qn}\left(X_{[0]}\right)$.

Proof. It suffices to show that ad $T_{H}\left(x_{i} x_{j}\right)$ is a nilpotent linear transformation of $W(n, n)_{[t]}$ for every $t \geq-1$. Suppose that $x^{(\alpha)} x^{u} D_{k}$ is a standard basis element of $W(m, n)_{[t]}$, where $t \geq-1$. To simplify our proof for the lemma, we only verify the case $i \in Y_{0}, j \in Y_{1}$, and $k \neq i, j$ as the proofs for the other cases are similar and hence omitted.

An induction on $l$ shows that $\left(\operatorname{ad} T_{H}\left(x_{i} x_{j}\right)\right)^{l}\left(x^{(\alpha)} x^{u} D_{k}\right)$

$$
=(-1)^{l-1} l x_{i}^{l-1} D_{j^{\prime}}^{l-1}\left(x^{(\alpha)}\right) x_{j} D_{i^{\prime}}\left(x^{u}\right) D_{k}+(-1)^{l} x_{i}^{l} D_{j^{\prime}}^{l}\left(x^{(\alpha)}\right) x^{u} D_{k}=0 .
$$

This yields that $\left(\operatorname{ad} T_{H}\left(x_{i} x_{j}\right)\right)^{l}\left(x^{(\alpha)} x^{u} D_{k}\right)=0$ when $l \geq t+3$. 
Let $I_{n}$ denote the identity matrix of size $2 n \times 2 n$, and let $e_{i j}$ denote the $2 n \times 2 n$ matrix whose $(i, j)$-entry is 1 and whose other entries are 0 . Set

$$
\begin{aligned}
& \tilde{p}(n)=\left\{\left(\begin{array}{cc}
A & B \\
C & -A^{\mathrm{T}}
\end{array}\right) \in \operatorname{gl}(n, n) \mid B=-B^{\mathrm{T}}, C=C^{\mathrm{T}}\right\}, \\
& p(n)=\left\{\left(\begin{array}{cc}
A & B \\
C & -A^{\mathrm{T}}
\end{array}\right) \in \tilde{p}(n) \mid \operatorname{tr} A=0\right\},
\end{aligned}
$$

where $A^{\mathrm{T}}$ is the transpose of $A$. Then $\tilde{p}(n), p(n)$ are subalgebras of $\operatorname{gl}(n, n)$ (see [Kac 1998]). Clearly,

$$
\begin{aligned}
& \tilde{p}(n)_{\overline{0}}=\left\{\left(\begin{array}{cc}
A & 0 \\
0 & -A^{\mathrm{T}}
\end{array}\right) \in \operatorname{gl}(n, n)\right\}, \\
& p(n)_{\overline{0}}=\left\{\left(\begin{array}{cc}
A & 0 \\
0 & -A^{\mathrm{T}}
\end{array}\right) \in \operatorname{gl}(n, n) \mid \operatorname{tr} A=0\right\} .
\end{aligned}
$$

Lemma 3.12. (1) $\rho\left(T_{H}\left(x_{i} x_{j}\right)\right)=(-1)^{\mu\left(i^{\prime}\right)} e_{i^{\prime} j}-(-1)^{\mu\left(i^{\prime}\right) \mu(j)} e_{j^{\prime} i}$ for all $i, j \in Y$, hence $\rho\left(H O_{[0]}\right)=\tilde{p}(n)$ and $\rho\left(H O_{[0]} \cap H O_{\overline{0}}\right)=\tilde{p}(n)_{\overline{0}}$.

(2) $\rho\left(T_{H}\left(x_{i} x_{j}\right)\right)=(-1)^{\mu\left(i^{\prime}\right)} e_{i^{\prime} j}-(-1)^{\mu\left(i^{\prime}\right) \mu(j)} e_{j^{\prime} i}$ for all $i, j \in Y$ with $i \neq j^{\prime}$, $\rho\left(T_{H}\left(x_{k} x_{k^{\prime}}-x_{l} x_{l^{\prime}}\right)\right)=e_{k k}-e_{k^{\prime} k^{\prime}}-e_{l l}+e_{l^{\prime} l^{\prime}}$ for all $k, l \in Y_{0}$ with $k \neq l$, hence $\rho\left(S H O_{[0]}\right)=p(n)$ and $\rho\left(S H O_{[0]} \cap S H O_{\overline{0}}\right)=p(n)_{\overline{0}}$.

Proof. (1) Direct calculation shows that ad $T_{H}\left(x_{i} x_{j}\right)\left(D_{j}\right)=(-1)^{\mu\left(i^{\prime}\right)} D_{i^{\prime}}$, and $\operatorname{ad} T_{H}\left(x_{i} x_{j}\right)\left(D_{i}\right)=-(-1)^{\mu\left(i^{\prime}\right) \mu(j)} D_{j^{\prime}}$. It follows that

$$
\rho\left(T_{H}\left(x_{i} x_{j}\right)\right)=(-1)^{\mu\left(i^{\prime}\right)} e_{i^{\prime} j}-(-1)^{\mu\left(i^{\prime}\right) \mu(j)} e_{j^{\prime} i} .
$$

Note that $H O_{0}=\operatorname{span}_{\mathbb{F}}\left\{T_{H}\left(x_{i} x_{j}\right) \mid i, j \in Y\right\}$. Consequently, (1) holds.

(2) The proof is similar to that of (1), hence omitted.

Lemma 3.13. $\rho\left(\operatorname{Qn}\left(X_{[0]} \cap X_{\overline{0}}\right)\right)=p(n)_{\overline{0}}$.

Proof. Suppose that $y \in \mathrm{qn}\left(X_{[0]} \cap X_{\overline{0}}\right)$. Then $\rho(y)$ is a nilpotent matrix by Lemma 3.8. It follows from Lemma 3.12 that $\rho(y)=\operatorname{diag}\left(A,-A^{\mathrm{T}}\right)$, where $A$ and $-A^{\mathrm{T}}$ are $n \times n$ nilpotent matrices. This shows that $\operatorname{tr} A=0$, that is $\rho(y) \in p(n)_{\overline{0}}$. Thus $\rho\left(\mathrm{qn}\left(X_{[0]} \cap X_{\overline{0}}\right)\right) \subseteq p(n)_{\overline{0}}$.

Conversely, set

$$
R=\left\{T_{H}\left(x_{i} x_{j}\right) \mid i \in Y_{0}, j \in Y_{1} \text {, with } i \neq j^{\prime}\right\} .
$$

Then $R \subseteq \mathrm{qn}\left(X_{[0]} \cap X_{\overline{0}}\right)$, by Lemma 3.11, whence $\rho(R) \subseteq \rho\left(\mathrm{qn}\left(X_{[0]} \cap X_{\overline{0}}\right)\right)$. Noting that $\rho\left(T_{H}\left(x_{i} x_{j}\right)\right)=e_{j^{\prime} i}-e_{i^{\prime} j}$ for all $i \in Y_{0}, j \in Y_{1}$ with $i \neq j^{\prime}$, by Lemma 3.12, we see that $\rho(R)$ generates $p(n)_{\overline{0}}$. Thus $p(n)_{\overline{0}} \subseteq \rho\left(\mathrm{Qn}\left(X_{[0]} \cap X_{\overline{0}}\right)\right)$.

Note that $\operatorname{Nor}_{X_{\overline{0}}}\left(\operatorname{Qn}\left(X_{\overline{0}}\right)\right)=\left\{y \in X_{\overline{0}} \mid\left[y, \operatorname{Qn}\left(X_{\overline{0}}\right)\right] \subseteq \operatorname{Qn}\left(X_{\overline{0}}\right)\right\}$. Clearly, the set $\operatorname{Nor}_{\overline{0}}\left(\mathrm{Qn}\left(X_{\overline{0}}\right)\right)$ is invariant under automorphisms of $X$. 
Proposition 3.14. $X_{0} \cap X_{\overline{0}}=\operatorname{Nor}_{X_{\overline{0}}}\left(\mathrm{Qn}\left(X_{\overline{0}}\right)\right)$. In particular, $X_{0} \cap X_{\overline{0}}$ is an invariant subalgebra.

Proof. Note that $\left[\tilde{p}(n)_{\overline{0}}, p(n)_{\overline{0}}\right]=p(n)_{\overline{0}}$. It follows from Lemmas 3.12 and 3.13 that

$$
\left[\rho\left(X_{[0]} \cap X_{\overline{0}}\right), \rho\left(\operatorname{Qn}\left(X_{[0]} \cap X_{\overline{0}}\right)\right)\right]=\left[\tilde{p}(n)_{\overline{0}}, p(n)_{\overline{0}}\right]=\rho\left(\operatorname{Qn}\left(X_{[0]} \cap X_{\overline{0}}\right)\right),
$$

whence

$$
\left[X_{[0]} \cap X_{\overline{0}}, \operatorname{Qn}\left(X_{[0]} \cap X_{\overline{0}}\right)\right]=\operatorname{Qn}\left(X_{[0]} \cap X_{\overline{0}}\right),
$$

since $\rho$ is faithful. By Lemma 3.10 and (11), we have

$$
\begin{aligned}
{\left[X_{0} \cap X_{\overline{0}}, \operatorname{Qn}\left(X_{\overline{0}}\right)\right] } & =\left[X_{[0]} \cap X_{\overline{0}}+X_{1} \cap X_{\overline{0}}, \operatorname{Qn}\left(X_{[0]} \cap X_{\overline{0}}\right)+X_{1} \cap X_{\overline{0}}\right] \\
& \subseteq\left[X_{[0]} \cap X_{\overline{0}}, \operatorname{Qn}\left(X_{[0]} \cap X_{\overline{0}}\right)\right]+X_{1} \cap X_{\overline{0}} \\
& \subseteq \operatorname{Qn}\left(X_{[0]} \cap X_{\overline{0}}\right)+X_{1} \cap X_{\overline{0}}=\operatorname{Qn}\left(X_{\overline{0}}\right) .
\end{aligned}
$$

Thus $X_{0} \cap X_{\overline{0}} \subseteq \operatorname{Nor}_{X_{\overline{0}}}\left(\mathrm{Qn}\left(X_{\overline{0}}\right)\right)$.

Conversely, suppose that $y=y_{-1}+y_{0} \in \operatorname{Nor}_{X_{\overline{0}}}\left(\operatorname{Qn}\left(X_{\overline{0}}\right)\right)$, where $y_{-1} \in X_{[-1]} \cap X_{\overline{0}}$, $y_{0} \in X_{0} \cap X_{\overline{0}}$. We want to show that $y_{-1}=0$. Assume $y_{-1}=\sum_{t=1}^{n} a_{t} D_{t} \neq 0$, where $a_{t} \in \mathbb{F}$. Then we can pick $k \in Y_{0}$ such that $a_{k} \neq 0$, and then pick $j_{k} \in Y_{1}$ such that $j_{k} \neq k^{\prime}$. From Lemma 3.11 we see that $T_{H}\left(x_{k} x_{j_{k}}\right) \in \mathrm{Qn}\left(X_{\overline{0}}\right)$, which combined with our hypothesis that $y \in \operatorname{Nor}_{X_{\overline{0}}}\left(\operatorname{Qn}\left(X_{\overline{0}}\right)\right)$, yields $\left[y, T_{H}\left(x_{k} x_{j_{k}}\right)\right] \in \operatorname{Qn}\left(X_{\overline{0}}\right)$, whence $\left[y, T_{H}\left(x_{k} x_{j_{k}}\right)\right] \in X_{0} \cap X_{\overline{0}}$ by Lemma 3.10. On the other hand, a direct calculation shows that

$\left[y, T_{H}\left(x_{k} x_{j_{k}}\right)\right]=\left[y_{-1}, T_{H}\left(x_{k} x_{j_{k}}\right)\right]+\left[y_{0}, T_{H}\left(x_{k} x_{j_{k}}\right)\right]=-a_{k} D_{j_{k}^{\prime}}+\left[y_{0}, T_{H}\left(x_{k} x_{j_{k}}\right)\right]$.

Since $\left[y_{0}, T_{H}\left(x_{k} x_{j_{k}}\right)\right] \in X_{0} \cap X_{\overline{0}}$, we see that $a_{k}=0$, contradicting our assumption, thus $y_{-1}=0$. So $y=y_{0} \in X_{0} \cap X_{\overline{0}}$, proving $\operatorname{Nor}_{X_{\overline{0}}}\left(\mathrm{Qn}\left(X_{\overline{0}}\right)\right) \subseteq X_{0} \cap X_{\overline{0}}$. Since $\operatorname{Nor}_{X_{\overline{0}}}\left(\mathrm{Qn}\left(X_{\overline{0}}\right)\right)$ is invariant, we see that $X_{0} \cap X_{\overline{0}}$ is invariant.

Set $\Omega=\left\{y \in \mathrm{qn}\left(X_{0} \cap X_{\overline{0}}\right) \mid\left[y, X_{0} \cap X_{\overline{0}}\right] \subseteq \mathrm{qn}\left(X_{0} \cap X_{\overline{0}}\right)\right\}$. Then Proposition 3.14 shows that $\Omega$ is invariant under automorphisms of $X$.

Proposition 3.15. $X_{1} \cap X_{\overline{0}}=\Omega$. In particular, $X_{1} \cap X_{\overline{0}}$ is an invariant subalgebra.

Proof. We only verify the case of $\mathrm{SHO}$, as the proof for $\mathrm{HO}$ is similar and hence omitted. Suppose that $y=y_{0}+y_{1}$ is an arbitrary element of $\Omega$, where $y_{0}$ is in $S H O_{[0]} \cap S H O_{\overline{0}}$ and $y_{1}$ is in $S H O_{1} \cap S H O_{\overline{0}}$. Suppose that

$$
y_{0}=\sum_{i, j \in Y_{0}, i \neq j} a_{i j} T_{H}\left(x_{i} x_{j^{\prime}}\right)+\sum_{i=1}^{n-1} a_{i i} T_{H}\left(x_{i} x_{i^{\prime}}-x_{i+1} x_{(i+1)^{\prime}}\right),
$$


where $a_{i j} \in \mathbb{F}$. Let $b_{i j}=a_{i j}$, where $i, j \in Y_{0}$ with $i \neq j$, and let $b_{i i}=a_{i i}-a_{i-1, i-1}$, where $i \in Y_{0}$, and $a_{00}=a_{n n}=0$. Let

$$
\begin{aligned}
& l=\min \left\{i \in Y_{0} \mid b_{i j_{0}} \neq 0 \text { for some } j_{0} \in Y\right\}, \\
& t=\min \left\{j \in Y_{0} \mid b_{i_{0} j} \neq 0 \text { for some } i_{0} \in Y\right\} .
\end{aligned}
$$

We first consider the case $l \leq t$. Set $k=\max \left\{j \in Y_{0} \mid b_{l j} \neq 0\right\}$. Then $l \leq t \leq k$, and $b_{l k} \neq 0$. If $l=k$, then

$$
y_{0}=\sum_{i=l+1}^{n} \sum_{j=l, i \neq j}^{n} a_{i j} T_{H}\left(x_{i} x_{j^{\prime}}\right)+\sum_{i=l}^{n-1} a_{i i} T_{H}\left(x_{i} x_{i^{\prime}}-x_{i+1} x_{(i+1)^{\prime}}\right),
$$

and

$$
\begin{aligned}
\rho\left(y_{0}\right)=\sum_{i=l}^{n} a_{i i}\left(e_{i i}-e_{i+1 i+1}\right)-\sum_{i=l}^{n} a_{i i}\left(e_{i^{\prime} i^{\prime}}-e_{(i+1)^{\prime}(i+1)^{\prime}}\right) & \\
& +\sum_{i=l+1}^{n} \sum_{j=l, i \neq j}^{n} a_{i j}\left(e_{j i}-e_{i^{\prime} j^{\prime}}\right)=\left(\begin{array}{cc}
a_{l l} A_{l l} & * \\
0 & *
\end{array}\right),
\end{aligned}
$$

where $A_{l l}$ is the $l \times l$ matrix whose $(l, l)$-entry is 1 and 0 elsewhere. Since $a_{l l} \neq 0$, we conclude that $\rho\left(y_{[0]}\right)$ is not a nilpotent matrix. It follows from Lemma 3.8 that $y \notin \mathrm{qn}\left(S H O_{\overline{0}}\right)$, contradicting the hypothesis that $y \in \Omega$. Therefore $l<k$ and

$$
y_{0}=\sum_{j=t, j \neq l}^{k} a_{l j} T_{H}\left(x_{l} x_{j^{\prime}}\right)+\sum_{i=l+1}^{n} \sum_{j=t, i \neq j}^{n} a_{i j} T_{H}\left(x_{i} x_{j^{\prime}}\right)+\sum_{i=t}^{n} a_{i i} T_{H}\left(x_{i} x_{i^{\prime}}-x_{i+1} x_{(i+1)^{\prime}}\right) .
$$

A direct calculation shows that

$$
\begin{aligned}
\rho\left(\left[T_{H}\left(x_{k} x_{l^{\prime}}\right), y_{0}\right]\right)= & {\left[e_{l k}-e_{k^{\prime} l^{\prime}}, \rho\left(y_{0}\right)\right] } \\
= & a_{l k}\left(e_{l l}-e_{l^{\prime} l^{\prime}}-e_{k k}+e_{k^{\prime} k^{\prime}}\right)-\sum_{j=t, j \neq l}^{k-1} a_{l j}\left(e_{j k}-e_{k^{\prime} j^{\prime}}\right) \\
& +\sum_{i=l+1, i \neq k}^{n} a_{i k}\left(e_{l i}-e_{i^{\prime} l^{\prime}}\right)+\left(a_{k k}-a_{k-1, k-1}-\delta_{l t} a_{l l}\right)\left(e_{l k}-e_{k^{\prime} l^{\prime}}\right) \\
= & \left(\begin{array}{rr}
a_{l k} A_{l l} & * \\
0 & *
\end{array}\right),
\end{aligned}
$$

so $\rho\left(\left[T_{H}\left(x_{k} x_{l^{\prime}}\right), y_{0}\right]\right)$ is not a nilpotent matrix. Since

$$
\left[T_{H}\left(x_{k} x_{l^{\prime}}\right), y\right]=\left[T_{H}\left(x_{k} x_{l^{\prime}}\right), y_{0}\right]+\left[T_{H}\left(x_{k} x_{l^{\prime}}\right), y_{1}\right],
$$

it follows from Lemma 3.8 that $\left[T_{H}\left(x_{k} x_{l^{\prime}}\right), y\right] \notin \mathrm{qn}\left(\mathrm{SHO}_{0}\right)$, contradicting our hypothesis that $y \in \Omega$. 
We now consider the case $l>t$. Set $k=\max \left\{i \in Y_{0} \mid b_{i t} \neq 0\right\}$. Then $t<l \leq k$, $b_{k t} \neq 0$ and $y_{0}=\sum_{i=l}^{k} a_{i t} T_{H}\left(x_{i} x_{t^{\prime}}\right)+\sum_{i=l}^{n} \sum_{j=t+1, i \neq j}^{n} a_{i j} T_{H}\left(x_{i} x_{j^{\prime}}\right)+\sum_{i=l}^{n} a_{i i} T_{H}\left(x_{i} x_{i^{\prime}}-x_{i+1} x_{(i+1)^{\prime}}\right)$. A direct computation shows that

$$
\begin{aligned}
\rho\left(\left[T_{H}\left(x_{t} x_{k^{\prime}}\right), y_{0}\right]\right)= & {\left[e_{k t}-e_{t^{\prime} k^{\prime}}, \rho\left(y_{0}\right)\right] } \\
= & -a_{k t}\left(e_{t t}-e_{t^{\prime} t^{\prime}}-e_{k k}+e_{k^{\prime} k^{\prime}}\right)+\sum_{i=l}^{k-1} a_{i t}\left(e_{k i}-e_{i^{\prime} k^{\prime}}\right) \\
& \quad-\sum_{j=t+1, j \neq k}^{n} a_{k j}\left(e_{j t}-e_{t^{\prime} j^{\prime}}\right)+\left(a_{k-1, k-1}-a_{k, k}\right)\left(e_{k t}-e_{t^{\prime} k^{\prime}}\right) \\
= & \left(\begin{array}{cc}
-a_{k t} B_{t t} & 0 \\
* & *
\end{array}\right),
\end{aligned}
$$

where $B_{t t}$ is the $t \times t$ matrix whose $(t, t)$-entry is 1 and 0 elsewhere. It follows that $\rho\left(\left[T_{H}\left(x_{t} x_{k^{\prime}}\right), y_{0}\right]\right)$ is not a nilpotent matrix, whence $\left[T_{H}\left(x_{t} x_{k^{\prime}}\right), y\right] \notin \mathrm{qn}\left(S H O_{0}\right)$ by Lemma 3.8, a contradiction which yields $y_{0}=0$. Therefore $y=y_{1} \in S H O_{1} \cap S H O_{\overline{0}}$, proving $\Omega \subseteq S H O_{1} \cap S_{H} O_{\overline{0}}$.

Conversely, noting that $\mathrm{SHO}_{1} \subseteq \mathrm{qn}(\mathrm{SHO})$, we see that

$\left[\mathrm{SHO}_{1} \cap \mathrm{SHO}_{\overline{0}}, \mathrm{SHO}_{0} \cap \mathrm{SHO}_{\overline{0}}\right]$

$$
\subseteq S H O_{1} \cap S H O_{\overline{0}} \subseteq S H O_{0} \cap S H O_{\overline{0}} \cap \mathrm{qn}(S H O)=\mathrm{qn}\left(S_{H} O_{0} \cap S H O_{\overline{0}}\right) .
$$

Thus $S H O_{1} \cap S H O_{\overline{0}} \subseteq \Omega$. Since $\Omega$ is invariant, we see that $S H O_{1} \cap S H O_{\overline{0}}$ is invariant.

Lemma 3.16. $\left[X_{\overline{1}}, X_{1} \cap X_{\overline{0}}\right]=X_{0} \cap X_{\overline{1}}$.

Proof. It suffices to show that $X_{0} \cap X_{\overline{1}} \subseteq\left[X_{\overline{1}}, X_{1} \cap X_{\overline{0}}\right]$. We first consider the case of $H O$. Suppose that $T_{H}\left(x^{(\alpha)} x^{u}\right) \in H O_{0} \cap H O_{\overline{1}}$, where $\alpha \in \mathbb{N}_{0}^{n}, u \in \mathbb{B}(n)$. Note that if $|u| \neq n$, then there exists $k \in Y_{1} \backslash\{u\}$ such that

$$
T_{H}\left(x^{(\alpha)} x^{u}\right)=\left[D_{k}, T_{H}\left(x^{(\alpha)} x_{k} x^{u}\right)\right] \in\left[H O_{\overline{1}}, H O_{1} \cap H O_{\overline{0}}\right],
$$

and if $|u|=n$, then

$$
-T_{H}\left(x^{(\alpha)} x^{u}\right)=\left[T_{H}\left(x_{i^{\prime}} x_{j}\right), T_{H}\left(x_{i} x^{(\alpha)} D_{j}\left(x^{u}\right)\right)\right] \in\left[H O_{\overline{1}}, H O_{1} \cap H O_{\overline{0}}\right],
$$

for all $i \in Y_{0}, j \in Y_{1}$.

Next, we consider the case of $\mathrm{SHO}$. Suppose that $\left[x^{(\alpha)}, x^{u}\right] \in \mathrm{SHO}_{0} \cap \mathrm{SHO}_{\overline{1}}$, where $\alpha \in \mathbb{N}_{0}^{n}, u \in \mathbb{B}(n)$. If $|u| \neq n$, then there exists $k \in Y_{1}$ such that $k \notin\{u\}$. It 
follows from (3) that

$$
-\left[x^{(\alpha)}, x^{u}\right]=\left[x_{k^{\prime}},\left[x^{(\alpha)}, x_{k} x^{u}\right]\right] \in\left[S H O_{\overline{1}}, S H O_{1} \cap S H O_{\overline{0}}\right] .
$$

If $|u|=n$, then by the hypothesis, we see that $|u|$ is even. It follows that

$$
\left[x^{(\alpha)}, x^{u}\right]=\left[x_{3^{\prime}} \cdots x_{n^{\prime}},\left[x^{\left(\alpha+\varepsilon_{3}\right)}, x_{3^{\prime}} x_{1^{\prime}} x_{2^{\prime}}\right]\right] \in\left[S H O_{\overline{1}}, S H O_{1} \cap S H O_{\overline{0}}\right] .
$$

Theorem 3.17. The natural filtration of $X$ is invariant under automorphisms of $X$.

Proof. By Proposition 3.15, we see that $X_{1} \cap X_{\overline{0}}$ is invariant under automorphisms of $X$. It follows from Lemma 3.16 that $X_{0} \cap X_{\overline{1}}$ is invariant, which combined with Proposition 3.14 yields that $X_{0}$ is invariant. Noting that

$$
X_{[i]}=\left\{y \in X \mid[y, X] \subseteq X_{[i-1]}\right\}
$$

for every $i \geq 1$, we see that $X_{[i]}$ is invariant. Thus $X$ is invariant.

\section{The automorphism group of $X$}

Lemma 4.1. Suppose that $\varphi_{X} \in$ Aut $X$. Then:

(1) $\varphi_{X}$ is a continuous automorphism.

(2) There exists an $\mathbb{F}$-basis $\left\{e_{1}, e_{2}, \ldots, e_{2 n}\right\}$ of $X_{[-1]}$ such that $\varphi_{X}\left(D_{i}\right) \equiv e_{i}(\bmod X)$.

Proof. (1) By Theorem 3.17, we have $\varphi_{X}\left(X_{i}\right) \subseteq X_{i}$ for every $i \geq-1$. It follows that $X_{i} \subseteq \varphi_{X}^{-1}\left(X_{i}\right)$. On the other hand, noting that $\varphi_{X}^{-1} \in$ Aut $X$, we see that $\varphi_{X}^{-1}\left(X_{i}\right) \subseteq X_{i}$. Consequently, $\varphi_{X}$ is a continuous automorphism.

(2) By Theorem 3.17, $\varphi_{X}$ can induce an $\mathbb{F}$-isomorphism $\bar{\varphi}_{X}$ of the quotient spaces

$$
\bar{\varphi}_{X}: X / X_{0} \rightarrow X / X_{0},
$$

such that $\bar{\varphi}_{X}\left(y+X_{0}\right)=\varphi_{X}(y)+X_{0}$ for all $y \in X$. Since $\left\{D_{i}+X_{0} \mid i \in Y\right\}$ is an $\mathbb{F}$-basis of $X / X_{0}$, it follows that $\left\{\varphi_{X}\left(D_{i}\right)+X_{0} \mid i \in Y\right\}$ is an $\mathbb{F}$-basis of $X / X_{0}$. Then for every $i \in Y$, there exists $e_{i} \in X_{[-1]}$ such that $\varphi_{X}\left(D_{i}\right)+X_{0}=e_{i}+X_{0}$. Thus (2) holds.

Proposition 4.2. Suppose that $\varphi, \psi \in$ Aut $X$. If $\left.\varphi\right|_{X_{[-1]}}=\left.\psi\right|_{X_{[-1]}}$, then $\varphi=\psi$.

Proof. We first use induction on $k$ to show that $\left.\varphi\right|_{X_{[k]}}=\left.\psi\right|_{X_{[k]}}$, where $k \geq-1$. The result is obvious for $k=-1$. We assume it for $k-1$. Suppose that $y \in X_{[k]}$, and let $z=\varphi(y)-\psi(y)$. Then

$$
\left[z, \psi\left(D_{i}\right)\right]=\left[\varphi(y)-\psi(y), \psi\left(D_{i}\right)\right]=\varphi\left(\left[y, D_{i}\right]\right)-\psi\left(\left[y, D_{i}\right]\right)=0
$$

for every $i \in Y$. By Lemma 4.1(2), we can write $\psi\left(D_{i}\right)=e_{i}+w_{i}$, where $\left\{e_{1}, \ldots, e_{2 n}\right\}$ is an $\mathbb{F}$-basis of $X_{[-1]}$ and $w_{i} \in X_{0}$. It follows that $\left[z, e_{i}+w_{i}\right]=0$. 
Suppose that $e_{i}=\sum_{j \in Y} a_{i j} D_{j}$, where $a_{i j} \in \mathbb{F}$, and let $\left(c_{i j}\right)_{1 \leq i, j \leq 2 n}=\left(a_{i j}\right)_{1 \leq i, j \leq 2 n}^{-1}$. Then $\left[z,-w_{i}\right]=\left[z, e_{i}\right]=\sum_{j \in Y} a_{i j}\left[z, D_{j}\right]$, whence

$$
\left[z, D_{l}\right]=\sum_{i=1}^{2 n} c_{l i}\left[z,-w_{i}\right] \quad \text { for all } l \in Y .
$$

Noting that $y \in X_{0}$, we see that $z \in X_{0}$. Thus we can write $z=\sum_{j \geq 0} z_{j}$, where $z_{j} \in X_{[j]}$. Applying (12), we have $\left[z, D_{l}\right] \in X_{0}$, thus $\left[z_{0}, D_{l}\right] \in X_{0} \cap X_{[-1]}=0$ for all $l \in Y$. This yields $z_{0}=0$. Now we can write $z=\sum_{j \geq 1} z_{j}$. Repeating the argument above, we can see that $z_{j}=0$ for each $j \geq 1$. It follows that $z=0$, whence $\left.\varphi\right|_{X_{[k]}}=\left.\psi\right|_{X_{[k]}}$. Hence $\varphi=\psi$, by Lemma 4.1 (1).

Given $\sigma \in$ Aut $\Lambda(n, n)$ and $D \in \operatorname{Der} \Lambda(n, n)$, we set $D^{\sigma}=\sigma D \sigma^{-1}$. Then $\tilde{\sigma}: D \mapsto D^{\sigma}$ is an automorphism of $\operatorname{Der} \Lambda(n, n)$. Let

$$
\operatorname{Aut}(\Lambda(n, n): X)=\{\sigma \in \operatorname{Aut} \Lambda(n, n)|| \tilde{\sigma}(X) \subseteq X\} .
$$

Then $\operatorname{Aut}(\Lambda(n, n): X)$ is a subgroup of Aut $\Lambda(n, n)$, and it is called the admissible automorphism group of $\Lambda(n, n)$ relative to $X$. Obviously, the morphism $\Phi: \operatorname{Aut}(\Lambda(n, n): X) \rightarrow$ Aut $X$ given by $\left.\sigma \mapsto \tilde{\sigma}\right|_{X}$ is a homomorphism of groups.

Lemma 4.3. (1) Suppose that $A \in \mathrm{M}_{2 n}(\Lambda(n, n))$. Then $\operatorname{pr}_{[0]}(A)$ is invertible if and only if $A$ is invertible.

(2) Suppose that $\left\{e_{1}, \ldots, e_{2 n}\right\}$ is a $\Lambda(n, n)$-basis of $W(n, n)$. Let $\operatorname{pr}_{[-1]}$ be the projection of $W(n, n)$ onto $W(n, n)_{[-1]}$. Then $\left\{\operatorname{pr}_{[-1]} e_{1}, \ldots, \operatorname{pr}_{[-1]} e_{2 n}\right\}$ is an $\mathbb{F}$ basis of $W(n, n)_{[-1]}$.

(3) Suppose that $\varphi$ is an automorphism of $X$, and suppose that $\left\{y_{i} \mid i \in Y\right\} \subset X$ is a $\Lambda(n, n)$-basis of $W(n, n)$. Then $\left\{\varphi\left(y_{i}\right) \mid i \in Y\right\}$ is also a $\Lambda(n, n)$-basis of $W(n, n)$.

(4) The natural filtration of $\Lambda(n, n)$ is invariant under automorphisms of $\Lambda(n, n)$.

Proof. (1) We first prove that $A$ is invertible when $\operatorname{pr}_{[0]} A$ is invertible. Set

$$
\begin{aligned}
& \mathrm{P}(n)_{1}=\left\{f \in \mathrm{P}(n) \mid \operatorname{pr}_{[0]}(f)=0\right\} \quad \text { and } \\
& T=\operatorname{span}_{\mathbb{F}}\left\{x^{(\alpha)} x^{u} \mid D_{i}\left(x^{u}\right) \neq 0 \text { for some } i \in Y\right\} .
\end{aligned}
$$

Then we can write $A=\operatorname{pr}_{[0]}(A)+B+C$, where $B \in \mathrm{M}_{2 n}\left(\mathrm{P}(n)_{1}\right), C \in \mathrm{M}_{2 n}(T)$. Let $D=\operatorname{pr}_{[0]}(A)+B$. Since $\mathrm{P}(n)$ is commutative, we see that det $D$ is well defined. Note that $\operatorname{pr}_{[0]}(\operatorname{det} D)=\operatorname{det}\left(\operatorname{pr}_{[0]} D\right) \neq 0$, we can write det $D=a+f$, where $0 \neq a \in \mathbb{F}, f \in \mathrm{P}(n)_{1}$. Put $g=a^{-1}\left(\sum_{i=0}^{\infty}(-1)^{i}\left(a^{-1} f\right)^{i}\right)$. A direct calculation shows that $g \operatorname{det} D=1$. It follows that $\operatorname{det} D$ is invertible, whence $D$ is invertible.

Let $E$ be the inverse of $D$. Since $C \in \mathrm{M}_{2 n}(T)$, we have $C E \in \mathrm{M}_{2 n}(T)$, which combined with the fact that the product of any $n+1$ elements of $T$ is 0 yields that 
$C E$ is nilpotent. Thus $I+C E$ is invertible. Consequently, we have

$$
A E(I+C E)^{-1}=(C+D) E(I+C E)^{-1}=(C E+D E)(I+C E)^{-1}=I .
$$

Therefore $A$ is invertible.

Using the fact that $\operatorname{pr}_{[0]}(A B)=\operatorname{pr}_{[0]}(A) \operatorname{pr}_{[0]}(B)$ for arbitrary matrices $A$ and $B$ in $\mathrm{M}_{2 n}(\Lambda(n, n))$, we can prove the converse implication.

(2) Suppose that $\left(D_{1}, \ldots, D_{2 n}\right)^{\mathrm{T}}=A\left(e_{1}, \ldots, e_{2 n}\right)^{\mathrm{T}}$, where $A \in \mathrm{M}_{2 n}(\Lambda(n, n))$. Then $\left(D_{1}, \ldots, D_{2 n}\right)^{\mathrm{T}}=\operatorname{pr}_{[0]} A\left(\operatorname{pr}_{[-1]}\left(e_{1}\right), \ldots, \operatorname{pr}_{[-1]}\left(e_{2 n}\right)\right)^{\mathrm{T}}$. Since $\left\{D_{1}, \ldots, D_{2 n}\right\}$ is an $\mathbb{F}$-basis of $W(n, n)_{[-1]}$, it follows that $\left\{\operatorname{pr}_{[-1]}\left(e_{1}\right), \ldots, \operatorname{pr}_{[-1]}\left(e_{2 n}\right)\right\}$ is an $\mathbb{F}$ basis of $W(n, n)_{[-1]}$.

(3) By Theorem 3.17, $\varphi$ induces canonically $\bar{\varphi} \in \operatorname{gl}\left(X / X_{0}\right)$. Denote by $\bar{y}_{i}$ the image of $y_{i}$ under the canonically map $X \rightarrow X / X_{0}$. Then $\left\{\bar{y}_{i} \mid i \in Y\right\}$ is an $\mathbb{F}$-basis of $X / X_{0}$. Assume that

$$
\left(\varphi\left(y_{1}\right), \ldots, \varphi\left(y_{2 n}\right)\right)^{\mathrm{T}}=A\left(D_{1}, \ldots, D_{2 n}\right)^{\mathrm{T}},
$$

where $A \in \mathrm{M}_{2 n}(\Lambda(n, n))$. Decomposing $A=\operatorname{pr}_{[0]} A+\operatorname{pr}_{1} A$, we obtain

$$
\left(\bar{\varphi}\left(\bar{y}_{1}\right), \ldots, \bar{\varphi}\left(\bar{y}_{2 n}\right)\right)^{\mathrm{T}}=\left(\overline{\varphi\left(y_{1}\right)}, \ldots, \overline{\varphi\left(y_{2 n}\right)}\right)^{\mathrm{T}}=\operatorname{pr}_{[0]} A\left(\bar{D}_{1}, \ldots, \bar{D}_{2 n}\right)^{\mathrm{T}} .
$$

This implies that $\operatorname{pr}_{[0]} A$ is invertible. It follows from (1) that $A$ is invertible. Therefore $\{\varphi(y) \mid i \in Y\}$ is a $\Lambda(n, n)$-basis of $W(n, n)$.

(4) Since Der $\Lambda(n, n)=W(n, n)$, we have Aut $\Lambda(n, n)=\operatorname{Aut}(\Lambda(n, n): W(n, n))$. By [Zhang and Liu 2004, Theorem 2.12], the natural filtration of $W(n, n)$ is invariant under Aut $W(n, n)$. Note that for every $i \in Y, \tilde{\sigma}\left(f D_{i}\right)=(\sigma f)\left(\tilde{\sigma} D_{i}\right)$, where $\sigma \in$ Aut $\Lambda(n, n)$ and $f \in \Lambda(n, n)$, which implies the desired result.

Theorem 4.4. The map $\Phi: \operatorname{Aut}(\Lambda(n, n): X) \rightarrow$ Aut $X$ given by $\left.\sigma \mapsto \tilde{\sigma}\right|_{X}$ is an isomorphism.

Proof. It suffices to show that $\Phi$ is bijective. Assume that $\sigma \in \operatorname{Aut}(\Lambda(n, n): X)$ is an element such that $\left.\tilde{\sigma}\right|_{X}=\left.1\right|_{X}$. We first use induction on $|\alpha|+|u|$ to show that $\sigma\left(x^{(\alpha)} x^{u}\right)=x^{(\alpha)} x^{u}$, where $x^{(\alpha)} x^{u}$ is a standard basis element of $\Lambda(n, n), \alpha \in \mathbb{N}_{0}^{n}$, $u \in \mathbb{B}(n)$. If $|\alpha|+|u|=1$, then $x^{(\alpha)} x^{u}=x_{i}$ for some $i \in Y$. Since for every $k \in Y$ $D_{k}\left(\sigma\left(x_{i}\right)\right)=\left(\tilde{\sigma}\left(D_{k}\right)\right)\left(\sigma\left(x_{i}\right)\right)=\sigma D_{k} \sigma^{-1} \sigma\left(x_{i}\right)=\sigma D_{k}\left(x_{i}\right)=\sigma\left(\delta_{i k}\right)=\delta_{i k}=D_{k}\left(\delta_{i k}\right)$, it follows that $D_{k}\left(\sigma\left(x_{i}\right)-x_{i}\right)=0$, which combined with Lemma 4.3(4) yields $\sigma\left(x_{i}\right)=x_{i}$. If $|\alpha|+|u|>1$, then by induction

$$
D_{k}\left(\sigma\left(x^{(\alpha)} x^{u}\right)-x^{(\alpha)} x^{u}\right)=\left(\tilde{\sigma} D_{k}\right) \sigma\left(x^{(\alpha)} x^{u}\right)-D_{k}\left(x^{(\alpha)} x^{u}\right)=0,
$$

for every $k \in Y$. Thus $\sigma\left(x^{(\alpha)} x^{u}\right)-x^{(\alpha)} x^{u} \in \mathbb{F} \cap \Lambda(n, n)_{1}=0$. Consequently, $\sigma=1$ and $\Phi$ is injective. 
We next prove that $\Phi$ is surjective. Suppose that $\varphi$ is in Aut $X$. Then by Lemma 4.3(3), $\left\{\varphi\left(D_{1}\right), \ldots, \varphi\left(D_{2 n}\right)\right\}$ is a $\Lambda(n, n)$-basis of $W(n, n)$. Therefore, we can suppose that $\varphi\left(T_{H}\left(x_{i} x_{j}\right)\right)=\sum_{t=1}^{2 n} h_{i j t} \varphi\left(D_{t}\right)$, where $i, j \in Y$ with $i \neq j^{\prime}$, and $h_{i j t} \in \Lambda(n, n)$. Applying Lemma $4.3(2)$, we have $h_{i j t} \in \Lambda(n, n)_{1}$. Thus

$$
\varphi\left(\left[D_{k}, T_{H}\left(x_{i} x_{j}\right)\right]\right)=\left[\varphi\left(D_{k}\right), \sum_{t=1}^{2 n} h_{i j t} \varphi\left(D_{t}\right)\right]=\sum_{t=1}^{2 n}\left(\varphi\left(D_{k}\right)\left(h_{i j t}\right)\right) \varphi\left(D_{t}\right) .
$$

On the other hand,

$$
\begin{aligned}
\varphi\left(\left[D_{k}, T_{H}\left(x_{i} x_{j}\right)\right]\right) & =\varphi\left[D_{k},(-1)^{\mu(i) \mu\left(j^{\prime}\right)} x_{j} D_{i^{\prime}}+(-1)^{\mu(j)} x_{i} D_{j^{\prime}}\right] \\
& =(-1)^{\mu(i) \mu\left(j^{\prime}\right)} \delta_{k j} \varphi\left(D_{i^{\prime}}\right)+(-1)^{\mu(j)} \delta_{k i} \varphi\left(D_{j^{\prime}}\right) .
\end{aligned}
$$

In particular, by letting $i=1$ and $j \in Y \backslash\left\{1^{\prime}\right\}$ in equations (13) and (14), one sees that $\varphi\left(D_{k}\right)\left(h_{1 j 1^{\prime}}\right)=\delta_{k j}+\delta_{k 1} \delta_{j 1}$ for all $k \in Y$. Similarly, by letting $i=2^{\prime}$ and $j=1^{\prime}$, we obtain $\varphi\left(D_{k}\right)\left(h_{2^{\prime} 1^{\prime} 2}\right)=\delta_{k 1^{\prime}}$. Let $h_{1}=\frac{1}{2} h_{111^{\prime}}, h_{1^{\prime}}=h_{2^{\prime} 1^{\prime 2}}$ and $h_{j}=h_{1 j 1^{\prime}}$ for $j \in Y \backslash\left\{1,1^{\prime}\right\}$. Then $h_{j} \in \Lambda(n, n)_{1}$ with $\operatorname{deg}\left(h_{j}\right)=\mu(j)$, and

$$
\varphi\left(D_{i}\right)\left(h_{j}\right)=\delta_{i j} \quad \text { for all } i, j \in Y .
$$

Suppose that $\varphi\left(D_{i}\right)=\sum_{t=1}^{2 n} f_{i t} D_{t}$, where $f_{i t} \in \Lambda(n, n)$. It follows from (15) that

$$
\left(\delta_{i j}\right)_{i, j \in Y}=\left(\varphi\left(D_{i}\right) h_{j}\right)_{i, j \in Y}=\left(f_{i j}\right)_{i, j \in Y}\left(D_{i} h_{j}\right)_{i, j \in Y} .
$$

This implies that $\left(D_{i} h_{j}\right)_{i, j \in Y}$ is invertible, whence $\left(\operatorname{pr}_{[0]}\left(D_{i} h_{j}\right)\right)_{i, j \in Y}$ is invertible by Lemma 4.3(1). Consequently, there exists $\sigma \in$ Aut $\Lambda(n, n)$ such that $\sigma\left(x_{i}\right)=h_{i}$ by Lemma 3.4, which combined with (15) yields

$$
\left(\tilde{\sigma} D_{i}-\varphi D_{i}\right)\left(h_{j}\right)=\sigma\left(D_{i} x_{j}\right)-\delta_{i j}=0
$$

for all $i, j \in Y$. Since $h_{1}, h_{2}, \ldots, h_{2 n}$ generate $\Lambda(n, n)$, we see that $\tilde{\sigma} D_{i}=\varphi D_{i}$, whence $\left.\tilde{\sigma}\right|_{X}=\varphi$ by Proposition 4.2.

\section{Acknowledgments}

This paper was completed when Q. Mu visited the College of William and Mary, and he thanks the university and Professor Junping Shi for their warm hospitality.

\section{References}

[Humphreys 1972] J. E. Humphreys, Introduction to Lie algebras and representation theory, Graduate Texts in Mathematics 9, Springer, New York, 1972. MR 48 \#2197 Zbl 0254.17004

[Jin 1992] N. Jin, "ad-nilpotent elements, quasi-nilpotent elements and invariant filtrations of infinite-dimensional Lie algebras of Cartan type”, Sci. China Ser. A 35:10 (1992), 1191-1200. MR 94j:17027 Zbl 0772.17007 
[Kac 1977] V. G. Kac, "Lie superalgebras", Advances in Math. 26:1 (1977), 8-96. MR 58 \#5803 Zbl 0366.17012

[Kac 1998] V. G. Kac, "Classification of infinite-dimensional simple linearly compact Lie superalgebras", Adv. Math. 139:1 (1998), 1-55. MR 99m:17006 Zbl 0929.17026

[Rudakov 1986] A. N. Rudakov, "Subalgebras and automorphisms of Lie algebras of Cartan type", Funktsional. Anal. i Prilozhen. 20:1 (1986), 83-84. In Russian; translated in Funct. Anal. Appl. 20 (1986), 72-73. MR 87m:17030 Zbl 0594.17015

[Scheunert 1979] M. Scheunert, The theory of Lie superalgebras, Lecture Notes in Mathematics 716, Springer, Berlin, 1979. MR 80i:17005 Zbl 0407.17001

[Strade and Farnsteiner 1988] H. Strade and R. Farnsteiner, Modular Lie algebras and their representations, Monographs and Textbooks in Pure and Applied Mathematics 116, Marcel Dekker, New York, 1988. MR 89h:17021 Zbl 0648.17003

[Wilson 1971] R. L. Wilson, "Classification of generalized Witt algebras over algebraically closed fields”, Trans. Amer. Math. Soc. 153 (1971), 191-210. MR 47 \#5070 Zbl 0218.17009

[Wilson 1975] R. L. Wilson, "Automorphisms of graded Lie algebras of Cartan type", Comm. Algebra 3:7 (1975), 591-613. MR 52 \#3259 Zbl 0318.17009

[Zhang 1997] Y. Zhang, "Finite-dimensional Lie superalgebras of Cartan type over fields of prime characteristic", Chinese Sci. Bull. 42:9 (1997), 720-724. MR 98h:17023 Zbl 0886.17022

[Zhang and Liu 2004] Y. Zhang and W. Liu, "The general and special superalgebras of formal vectorfields”, Sci. China Ser. A 47:2 (2004), 272-283. MR 2005g:17040 Zbl 1124.17304

Received September 22, 2011. Revised October 27, 2011.

\section{REN}

SCHOOL OF MATHEMATICS AND STATISTICS

NORTHEAST NORMAL UNIVERSITY

CHANGCHUN, 130024

CHINA

ren1944@nenu.edu.cn

QIANG MU

SCHOOL OF Mathematical SCIENCES

HARBIN NORMAL UNIVERSITY

HARBIN, 150025

CHINA

qmu520@gmail.com

YONGZHENG ZHANG

SCHOOL OF MATHEMATICS AND STATISTICS

NORTHEAST NORMAL UNIVERSITY

ChangChun, 130024

CHINA

zhyz@nenu.edu.cn 


\title{
PACIFIC JOURNAL OF MATHEMATICS
}

\author{
http://pacificmath.org \\ Founded in 1951 by \\ E. F. Beckenbach (1906-1982) and F. Wolf (1904-1989)
}

\section{EDITORS}

V. S. Varadarajan (Managing Editor)

Department of Mathematics

University of California

Los Angeles, CA 90095-1555

pacific@math.ucla.edu

Vyjayanthi Chari

Department of Mathematics

University of California

Riverside, CA 92521-0135

chari@math.ucr.edu

\section{Robert Finn}

Department of Mathematics Stanford University

Stanford, CA 94305-2125

finn@math.stanford.edu

Kefeng Liu

Department of Mathematics

University of California

Los Angeles, CA 90095-1555

liu@math.ucla.edu
Darren Long

Department of Mathematics

University of California

Santa Barbara, CA 93106-3080

long@math.ucsb.edu

Jiang-Hua Lu

Department of Mathematics

The University of Hong Kong

Pokfulam Rd., Hong Kong jhlu@maths.hku.hk

Alexander Merkurjev

Department of Mathematics

University of California

Los Angeles, CA 90095-1555

merkurev@math.ucla.edu
Sorin Popa

Department of Mathematics University of California

Los Angeles, CA 90095-1555 popa@math.ucla.edu

Jie Qing

Department of Mathematics

University of California

Santa Cruz, CA 95064

qing@cats.ucsc.edu

Jonathan Rogawski

Department of Mathematics

University of California

Los Angeles, CA 90095-1555

jonr@math.ucla.edu

\section{PRODUCTION}

pacific@math.berkeley.edu

\section{SUPPORTING INSTITUTIONS}

ACADEMIA SINICA, TAIPEI

CALIFORNIA INST. OF TECHNOLOGY INST. DE MATEMÁTICA PURA E APLICADA KEIO UNIVERSITY

MATH. SCIENCES RESEARCH INSTITUTE NEW MEXICO STATE UNIV.

OREGON STATE UNIV.

\author{
STANFORD UNIVERSITY \\ UNIV. OF BRITISH COLUMBIA \\ UNIV. OF CALIFORNIA, BERKELEY \\ UNIV. OF CALIFORNIA, DAVIS \\ UNIV. OF CALIFORNIA, LOS ANGELES \\ UNIV. OF CALIFORNIA, RIVERSIDE \\ UNIV. OF CALIFORNIA, SAN DIEGO \\ UNIV. OF CALIF., SANTA BARBARA
}

\author{
UNIV. OF CALIF., SANTA CRUZ \\ UNIV. OF MONTANA \\ UNIV. OF OREGON \\ UNIV. OF SOUTHERN CALIFORNIA \\ UNIV. OF UTAH \\ UNIV. OF WASHINGTON \\ WASHINGTON STATE UNIVERSITY
}

These supporting institutions contribute to the cost of publication of this Journal, but they are not owners or publishers and have no responsibility for its contents or policies.

See inside back cover or pacificmath.org for submission instructions.

The subscription price for 2012 is US \$420/year for the electronic version, and \$485/year for print and electronic.

Subscriptions, requests for back issues from the last three years and changes of subscribers address should be sent to Pacific Journal of Mathematics, P.O. Box 4163, Berkeley, CA 94704-0163, U.S.A. Prior back issues are obtainable from Periodicals Service Company, 11 Main Street, Germantown, NY 12526-5635. The Pacific Journal of Mathematics is indexed by Mathematical Reviews, Zentralblatt MATH, PASCAL CNRS Index, Referativnyi Zhurnal, Current Mathematical Publications and the Science Citation Index.

The Pacific Journal of Mathematics (ISSN 0030-8730) at the University of California, c/o Department of Mathematics, 969 Evans Hall, Berkeley, CA 94720-3840, is published monthly except July and August. Periodical rate postage paid at Berkeley, CA 94704, and additional mailing offices. POSTMASTER: send address changes to Pacific Journal of Mathematics, P.O. Box 4163, Berkeley, CA 94704-0163.

PJM peer review and production are managed by EditFLOW ${ }^{\mathrm{TM}}$ from Mathematical Sciences Publishers.

PUBLISHED BY PACIFIC JOURNAL OF MATHEMATICS

at the University of California, Berkeley 94720-3840

A NON-PROFIT CORPORATION

Typeset in LATEX

Copyright $(02012$ by Pacific Journal of Mathematics 


\section{PACIFIC JOURNAL OF MATHEMATICS}

Volume $257 \quad$ No. $2 \quad$ June 2012

Extending triangulations of the 2-sphere to the 3-disk preserving a

4-coloring

RUi PEDRO CARPENTIER

Orthogonal quantum group invariants of links

LIN CHEN and QINGTAO CHEN

Some properties of squeezing functions on bounded domains

FUSHENG DENG, QIAN GUAN and LIYOU ZHANG

Representations of little $q$-Schur algebras

JiE DU, QIANG FU and JiAN-PAN WANG

Renormalized weighted volume and conformal fractional Laplacians

MARÍA DEL MAR GONZÁLEZ

The $L_{4}$ norm of Littlewood polynomials derived from the Jacobi symbol 395

JONATHAN JEDWAB and KAI-UWE SCHMIDT

On a conjecture of Kaneko and Ohno

ZHONG-HUA LI

Categories of unitary representations of Banach-Lie supergroups and restriction functors

StéPhane Merigon, Karl-Hermann NeEb and Hadi

SALMASIAN

Odd Hamiltonian superalgebras and special odd Hamiltonian superalgebras of formal vector fields

Li Ren, Qiang Mu and YongZheng ZHANG

Interior derivative estimates for the Kähler-Ricci flow

Morgan SHERMAN and BEN WEINKOVE

Two-dimensional disjoint minimal graphs

LINFENG ZHOU 